\title{
Coupled motion of cervical spine in three level hybrid constructs
}

\author{
Zhenhua Liao ${ }^{\mathrm{a}, \mathrm{b}}$, Ting $\mathrm{Pu}^{\mathrm{c}}$, Hongsheng $\mathrm{Gu}^{\mathrm{d}}$ and Weiqiang Liu ${ }^{\mathrm{a}, \mathrm{b}, *}$ \\ ${ }^{a}$ Department of Mechanical Engineering, Tsinghua University, Beijing 100084, China \\ ${ }^{b}$ Biomechanics and Biotechnology Lab, Research Institute of Tsinghua University in Shenzhen, \\ Shenzhen 518057, China \\ ${ }^{c}$ Machinery technology development CO LTD, Beijing 100044, China \\ ${ }^{d}$ Shenzhen Second Hospital, Shenzhen 518039, China
}

\begin{abstract}
To compare the biomechanical characters of three-level anterior cervical fusion and hybrid constructs by measuring coupled motion changes of the cervical spine, Eighteen adult human cadaveric cervical spines were biomechanically investigated under eccentric displacement control in lateral bending and axial rotation by measuring vertebral motion (X, Y, Z -axis). The 3DPD condition displayed similar coupled motion compared to the intact condition both in lateral bending and in axial rotation, while the 3PDP condition was similar only in lateral bending. However, the coupled motion of the cervical spine under 3P conditions markedly changed in both lateral bending and axial rotation. Considering the coupled motion characteristics, the 3DPD hybrid construct is biomechanically advantageous in three types of reconstructions (3DPD, 3PDP, 3P).
\end{abstract}

Keywords: Cervical spine, coupled motion, hybrid surgery, fusion, cervical disc replacement

\section{Introduction}

As a standard procedure, anterior cervical discectomy and fusion (ACDF) has been the most widely accepted procedure for the surgical therapy of cervical spondylosis, with satisfactory clinical outcomes [1]. However, by altering the normal biomechanics of the cervical spine, ACDF notably decreases mobility at the fused levels and increases motion at the adjacent levels, particularly in the presence of multilevel cervical disease. Although ACDF has achieved satisfactory effects in the short term, the increasing biomechanical stress on adjacent levels may result in the acceleration of adjacent segment degeneration (ASD) and the requirement of further surgery [2].

Recently, artificial cervical disc replacement (ACDR) has been progressively recommended as an alternative technique for ACDF. ACDR is designed to preserve motion and to reproduce the physiological kinematics of the cervical spine, which may reduce stress on adjacent discs and prevent subsequent ASD [3]. Although both laboratory research and clinical studies have demonstrated the safety and efficacy of ACDR at a single level [4], multilevel cervical degenerative disc diseases are

\footnotetext{
* Address for Correspondence: Weiqiang Liu, Research Institute of Tsinghua University in Shenzhen, Shenzhen 518057 , China. Tel.: +86 0755 26551376; Fax: +86 0755 26551380; E-mail: weiqliu@hotmail.com.
} 
common in clinical practice. Considering that ACDF involved more fused levels, it may lead to a greater loss of mobility in operative levels, and ACDR may provide benefits to adjacent segments. However, strict indications and hypermobility of the operative levels may limit the application of multilevel ACDR.

Given this tradeoff, Hybrid surgery (HS) which incorporates ACDF and ACDR at different levels may be the ideal surgical technique for the preservation of motion of operative levels, and avoiding long-level fusion. HS may therefore be considered as a treatment to balance the advantages and disadvantages of fusion and non-fusion techniques [5].

However, as an emerging procedure, the appropriate experimental evidence to support HS is still lacking. There are a few in vitro biomechanical studies and clinical reports comparing two-level ACDR versus arthrodesis and a hybrid construct, but little research has been done to compare the biomechanical behaviors of three-level standard anterior cervical arthrodesis and a hybrid construct [6]. The objective of this experimental study was to analyze the biomechanical characteristics after threelevel ACDF and hybrid surgery by depicting the main and coupled motions of cervical vertebra.

\section{Materials and methods}

\subsection{Specimen preparation}

Eighteen fresh adult human cadaveric cervical spines (C2-T1; age range, 52-73 years) were utilized for biomechanical testing. All cervical spines were evaluated for bone mineral density (BMD) using dual-energy x-ray absorptiometry scanning; measured BMD values ranged from 0.53 to $0.72 \mathrm{~g} / \mathrm{cm}^{2}$. Ligamentous structures were preserved while the musculature and fascia were carefully removed. Cervical spines with degenerative diseases or traumatic pathology were excluded by anteroposterior and lateral screening radiographs before biomechanical testing. Once harvested, all cervical specimens were immediately conserved in plastic bags and frozen at $-20^{\circ} \mathrm{C}$. In preparation for biomechanical testing, the required spines were thawed at $4^{\circ} \mathrm{C}$ for 12 hours. At room temperature on the testing day, the proximal vertebra (C2) and distal vertebra (T1) were separately mounted in a cylindrical container using a low fusion point $\left(72^{\circ} \mathrm{C}\right)$ alloy. Next, the $\mathrm{C} 2$ container was attached to the upper fixture while the $\mathrm{T} 1$ container was mounted to the lower testing platform. All tested cervical spines were mounted in neutral upright orientation. Markers made of four plexiglass motion detectors were fixed to the anterior aspects of each vertebra from $\mathrm{C} 2$ to T1.These markers can be detected by an optoelectronic motion measurement system. The three-dimensional range of motion of each vertebra was obtained with an optoelectronics measurement system (Optotrak, Northern Digital Inc., Waterloo, Ontario, Canada) capable of capturing the motion curve of the markers.

\subsection{Biomechanical test protocol}

Biomechanical tests were performed under displacement control by MTS machine (CMT6104; MTS Systems (China) Corp., Shenzhen, China) [7]. The flexion-extension axis of each tested spine was placed eccentric to the load axis of the actuator. Flexion-extension bending moments and a compressive load were applied to the upper container. The bending moments were limited to the upper bound of physiological human bending $(4.5 \mathrm{~nm})$. An angular displacement transducer was assembled to measure the global rotation of the cervical spine $(\mathrm{C} 2-\mathrm{T} 1)$. A displacement transducer was used to measure the changes in moment arm length between the upper container and the load axis of the MTS 
machine. All testing specimens were preconditioned by the application of three loading cycles. The spine specimens were tested by application of a $50 \mathrm{~N}$ compressive preload in flexion-extension and lateral bending circumstances. During the biomechanical tests, all specimens were moistened with $0.9 \% \mathrm{NaCl}$ physiologic serum spray to avoid tissue dehydration.

The purpose of this study was to evaluate the biomechanical properties of the cervical spine in three dimensions. Therefore, a three-dimensional coordinate system was defined. The posterior-inferior corner of each cervical vertebra was defined as the origin. The positive y axis was determined along the posterior wall of the inferior vertebra body, pointing upward. The positive $\mathrm{z}$ axis was perpendicular to the $\mathrm{y}$ axis, pointing forward. The positive $\mathrm{x}$ axis was perpendicular to both the $\mathrm{y}$ and $\mathrm{z}$ axes, pointing left.

\subsection{Reconstruction procedures}

First, the intact spines were analyzed. Each specimen was then reconstructed at C3-C6 (three-level) motion segments with the following treatments (Figure 1): 1) C3-C4 anterior cervical disc replacement (ACDR), C4-C5 anterior cervical plate (ACP), C5-C6 ACDR, (three-level disc plate disc, 3DPD); 2) C3-C4 ACP, C4-C5 ACDR, C5-C6 ACP (three-level plate disc plate, 3PDP); 3) C3-C6 ACP (threelevel plate, 3P). Measurements included vertebral motion (X, Y, Z-axis), applied load and moment.

The ACDR consisted of a titanium-ceramic alloy that fit between the top and bottom surfaces of the neck bones (Prestige LP Cervical Disc, Medtronic Sofamor Danek USA, Inc.). The arthrodesis was performed using an interbody PEEK cage (Telamon TM, Medtronic Sofamor Danek USA, Inc.) combined with an anterior cervical plating (ACP) system (DOC Cervical Plate, Depuy Spine, Inc., Raynham, MA, USA) as shown in Figure 1.

\section{Results}

In clinical practice, it is well known that two kinds of coupled motions exit in the cervical spine: axial rotation in the same direction as the lateral bending, and lateral bending in the same direction as the axial rotation, both of which were experimentally applied [8]. Therefore, this test was used to determine if the cervical spine could recover nearly normal coupled motion after the three-level

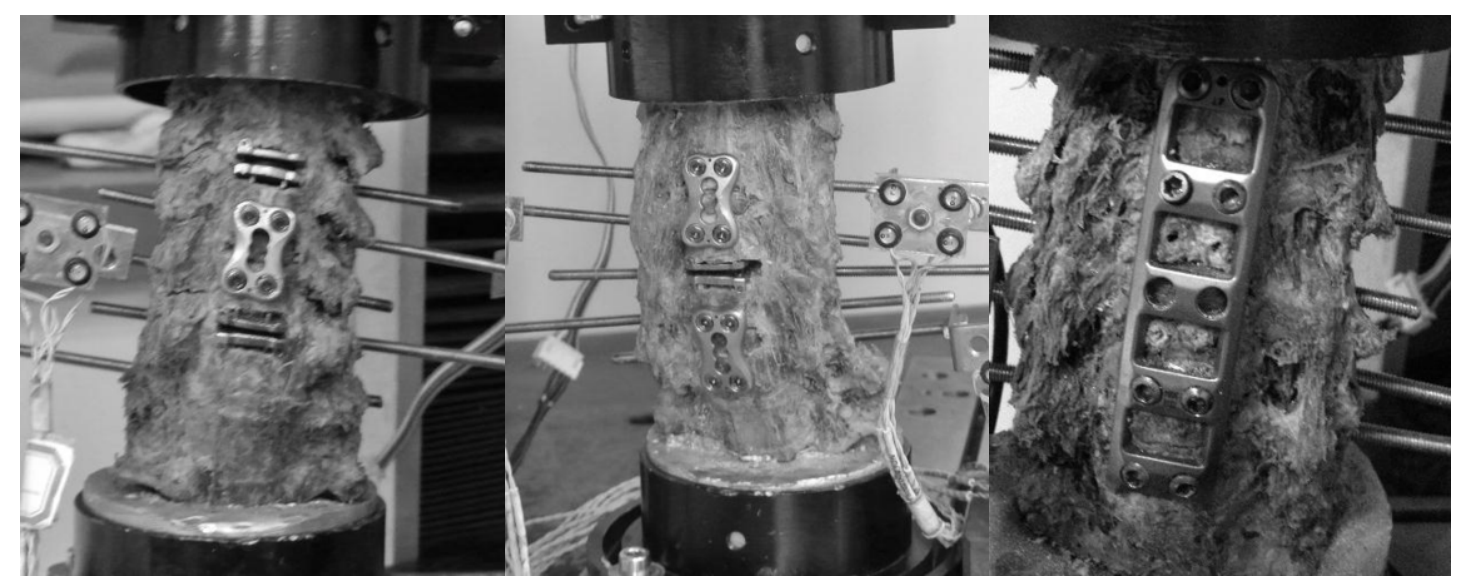

Fig. 1. Cervical specimen after surgery (left: 3DPD, middle: 3PDP, right: 3P). 
anterior plate fusion (3P), the 3PDP and 3DPD conditions, especially in regard to lateral bending and axial rotation.

\subsection{Lateral bending}

In lateral bending (left and right), the 3DPD and 3PDP conditions did not significantly change the regularity of normal coupled motion compared to the intact condition. In an intact condition, remarkable axial rotation coupled in the same direction was observed as the lateral bending moment was applied, whereas a 35\% rotation around the y-axis existed in the intact condition. Despite the slight decline in the significance of coupled motion, as the lateral bending moment was applied, axial rotation coupled in the same direction occurred at all intersegmental segments in the 3DPD and 3PDP conditions, whereas a $30 \%$ rotation around the y-axis was observed in the 3DPD condition and a $20 \%$ rotation was observed in the 3PDP condition. However, the regularity of coupled motion varied significantly in the 3P condition, which did not display nearly normal coupled motion as compared to an intact specimen (Figure 2). Alternatively, the 3DPD and 3PDP conditions did not display significant increases in motion change in the adjacent segments, while the $3 \mathrm{P}$ condition did.

\subsection{Axial rotation}

In left and right axial rotation, the 3DPD condition did not significantly change the regularity of
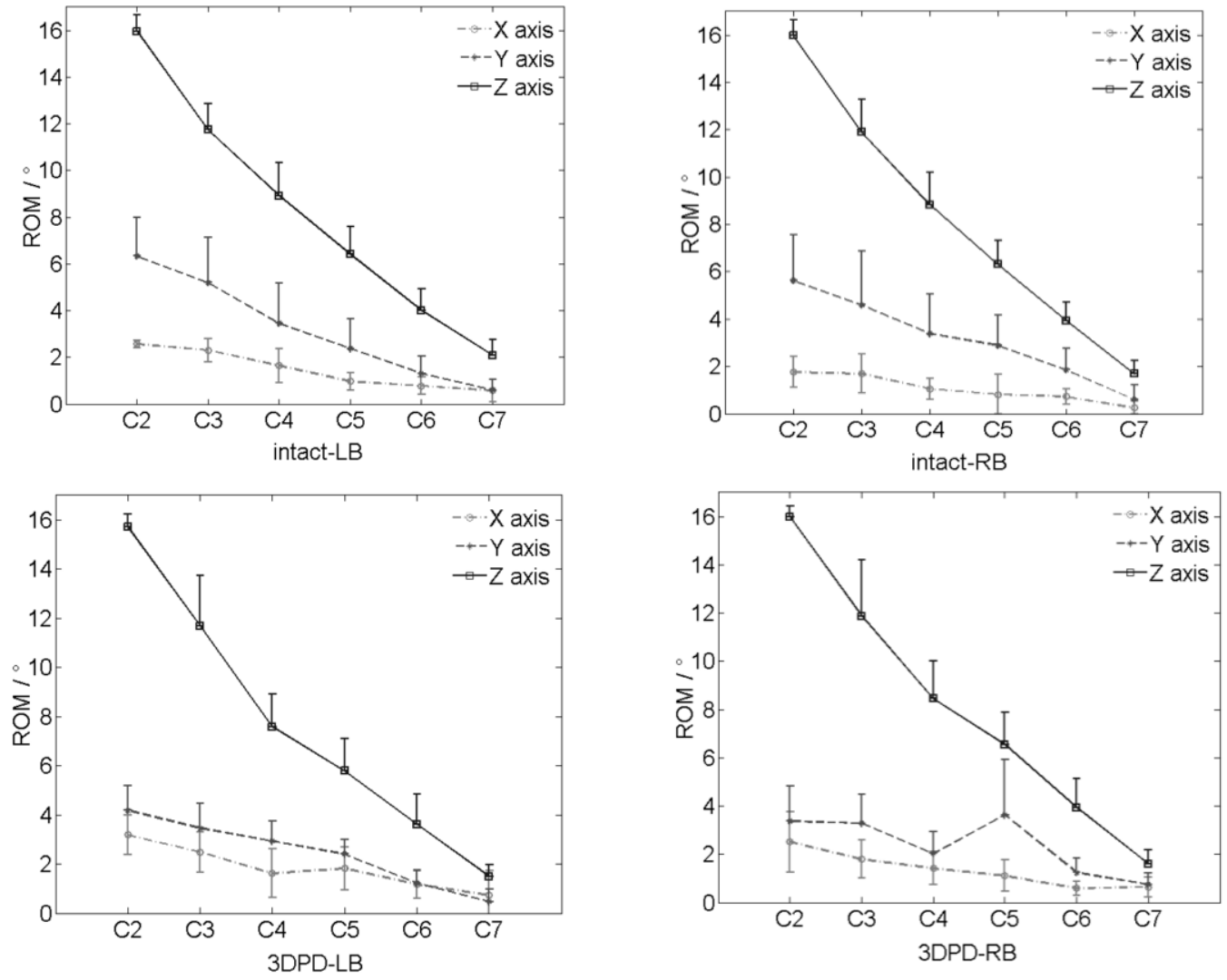

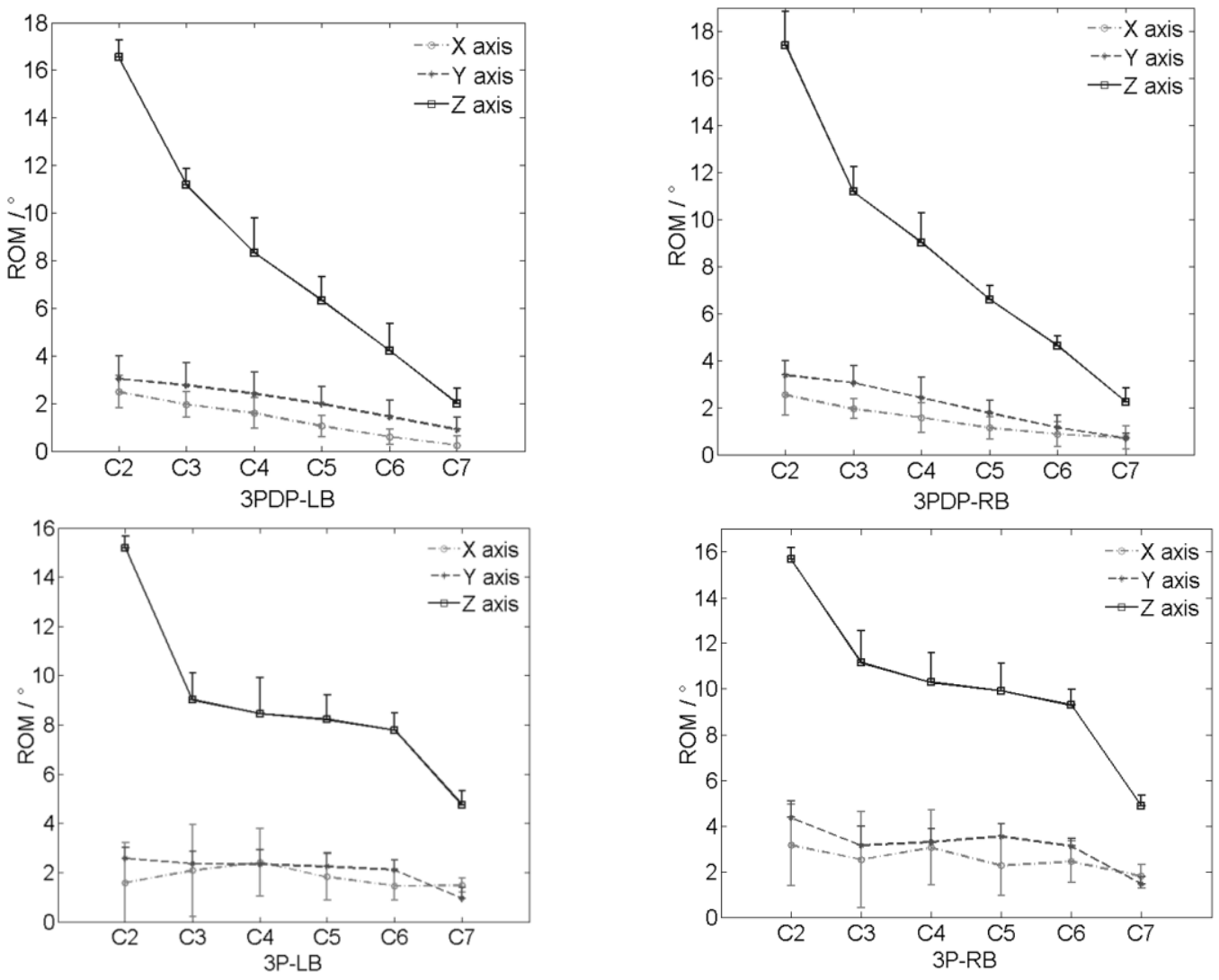

Fig. 2. Rotation angles of each segment around $\mathrm{X} / \mathrm{Y} / \mathrm{Z}$ axis under intact, 3DPD, 3PDP, and 3P conditions in lateral bending (LB: left bending; RB: right bending).

normal coupled motion compared to the intact condition. The axial rotation moment elicited mild coupled lateral bending and flexion-extension in the same direction as the axial rotation; a $13 \%$ lateral bending around the z-axis and a 13\% flexion-extension around the s-axis were observed in the intact condition. The 3DPD condition demonstrated coupled motion which was similar to the intact condition. However, the regularity of coupled motion varied significantly in the 3PDP and 3P
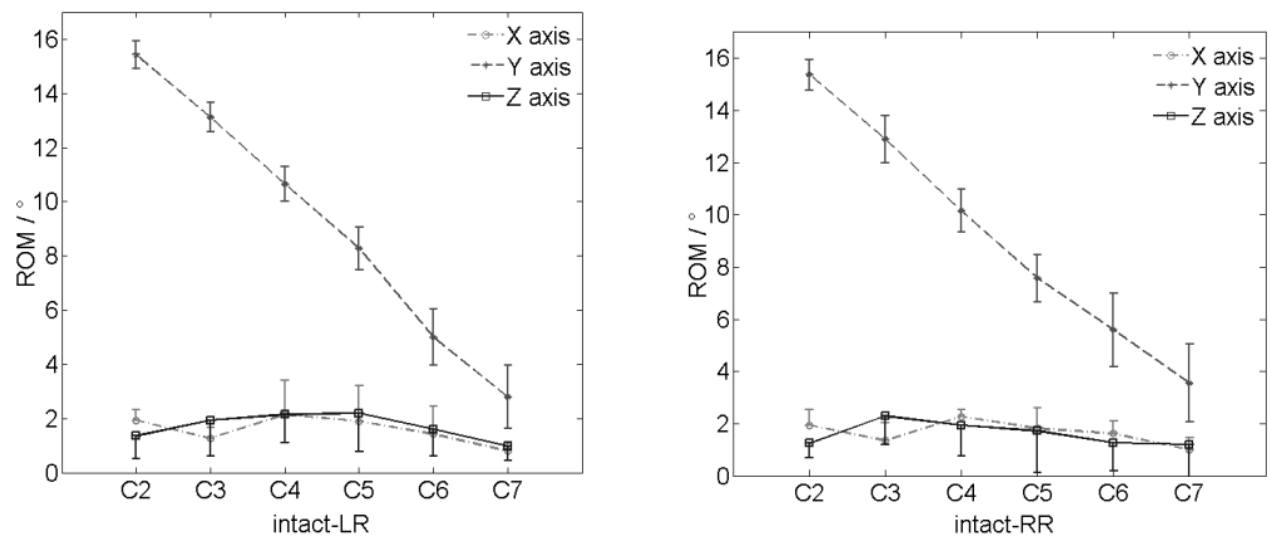

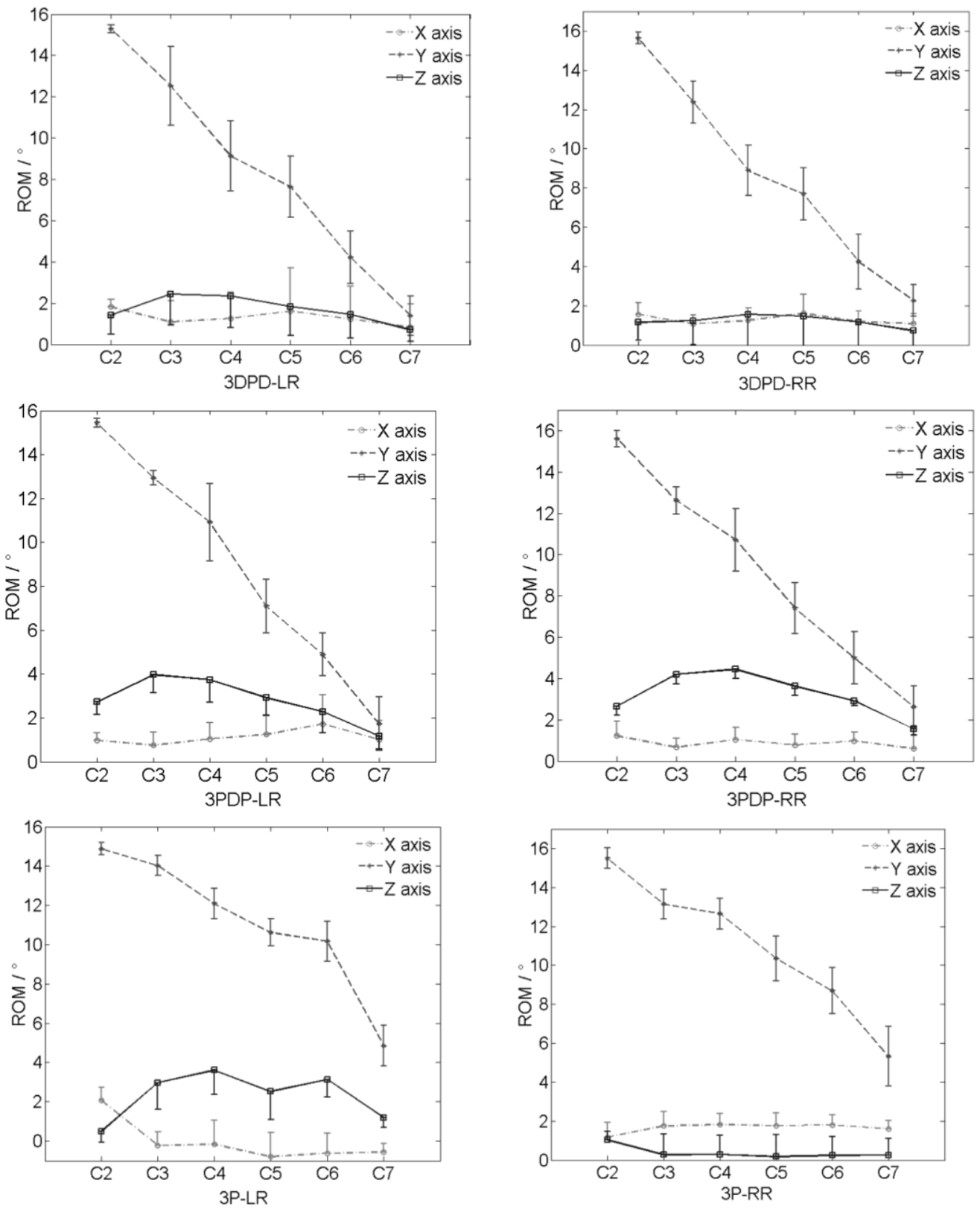

Fig. 3. Rotation angles of each segment around $\mathrm{X} / \mathrm{Y} / \mathrm{Z}$ axis under intact, 3DPD, 3PDP, 3P conditions in axial rotation (LR: left rotation; RR: right rotation).

conditions, which did not display normal coupled motion (Figure 3).

\section{Discussion}


Hybrid surgery has become one of the most controversial subjects in clinical spine technology. The present biomechanical study shows that HS could maintain segmental motion of the operative levels and have less adverse influence on adjacent levels. Therefore, HS may be a safe and effective alternative for multilevel cervical degenerative disc diseases [9].

The results of current biomechanical research are still primarily relevant to two-level hybrid surgery. These studies [10-14] indicate that two-level HS could preserve the motion of the treated levels and prevent overload of the adjacent discs and subsequent adjacent segment degeneration. In contrast to HS, two-level fusion largely constrains the motion of operative levels and induces compensatory increase in motion at adjacent levels, which may adversely increase the probability of adjacent segment degeneration. From a single surgeon series, Cardoso, et al. [15] presented HS as a safe and effective alternative to multilevel fusion for thirty-one patients ( 24 patients received a two-level hybrid construct, and 7 patients received a three-level hybrid construct), with an average clinical and radiological follow-up duration of 18 months. Hey, et al. [9] provided a direct comparison of all three groups with a minimum follow-up of two years. Seven consecutive patients who underwent HS were matched with another seven patients who underwent ACDF or ACDR based on levels of surgery. They found that HS appears comparable to ACDR and ACDF in terms of feasibility and safety, and that patients demonstrated an average earlier return to work. Thus, HS seems an attractive choice if patients were carefully selected.

However, biomechanical research focused on three-level hybrid surgery is still rare. Despite clinical findings [16] which suggest that hybrid surgery is an effective and safe option for cervical disk disease involving three levels, the overall quality of evidence for biomechanical characteristics of three-level HS is low. The primary concern regarding three-level hybrid constructs is the spinal kinematics, including the total range of motion (ROM) of the entire test cervical segment, as well as the ROM at the operative and adjacent levels [6]. The coupled motion of the cervical spine after hybrid surgery was seldom investigated.

However, considering the greater complexity of the three-level HS, not only the motion of instrumented levels and adjacent levels but also the coupled motion of the cervical spine after threelevel HS should be investigated. As one of the most important kinematic characters of cervical spine $[8,17]$, the coupled motion of the cervical spine after three-level HS should ideally be similar to the intact condition. This paper compared the coupled motion of the described 3DPD, 3PDP and 3P conditions to the intact condition in lateral bending and axial rotation.

This study presented that the most successful lateral bending occurred in the superior region primarily at $\mathrm{C} 2-\mathrm{C} 4$ as the lateral bending moment was applied in the intact condition. The lateral bending coupled with axial rotation in the same direction existed at all intersegmental segments; there were also coupled extensions at C2-C5. In contrast, both the 3DPD and 3PDP conditions retained similar coupled motion compared to the intact condition in lateral bending, while the 3P condition did not. In axial rotation, coupled axial rotation and extension were not observable in the intact condition; the 3DPD condition retained similar coupled motion compared to the intact condition, while the 3PDP and $3 \mathrm{P}$ conditions did not.

In general, the 3DPD condition retained similar coupled motion compared to the intact condition both in lateral bending and in axial rotation, and the 3PDP condition only retained similar coupled motion compared to the intact condition in lateral bending. Alternatively, the coupled motion of the cervical spine with the $3 \mathrm{P}$ condition markedly changed compared to the intact condition both in lateral bending and in axial rotation.

However, especially in lateral bending, the $3 \mathrm{P}$ condition led to exaggerated contribution of adjacent segments to global ROM compared to the intact spine. This phenomenon may potentially give rise to 
accelerated adjacent segment disease (ASD). On the contrary and as expected, although the 3DPD and 3PDP conditions restored only partial cervical kinematics at instrumented levels, both hybrid constructs caused only minimal changes to ROM at adjacent levels. This confirmed the finding that hybrid constructions could avoid huge motion increases in adjacent levels and may reduce the risk of ASD.

A limitation of the current study was the lack of intradiscal pressure (IDP) measurements. If pressure sensors were used in adjacent discs, the changes in IDP could be measured during the testing, which should contribute to further understanding of ASD. An additional limitation was the inability to verify the facet load using a finite element model and study the effects of disc degeneration.

\section{Conclusion}

This study analyzed the coupled motion characteristics of the cervical spine after 3DPD, 3PDP and 3P conditions were applied to human cervical specimens. These biomechanical findings support that the 3DPD condition could commendably retain normal coupled motion compared to the intact condition while the 3PDP and 3P conditions could not. In conclusion, the 3DPD hybrid construct may be biomechanically advantageous for three-level cervical reconstructions.

\section{Acknowledgment}

This project is supported by the National Key Technology R \& D Program of China (Grant No. 2012BAI18B05) and Technology Innovation Program of Development Special Fund of Shenzhen Strategic Emerging Industry (Grant No. JSGG20130624162316425).

\section{References}

[1] J.K. Burkus and R.W. Haid, Long-term clinical and radiographic outcomes of cervical disc replacement with the Prestige disc: Results from a prospective randomized controlled clinical trial, Journal of Neurosurgery Spine 13 (2010), 308-318.

[2] W.H.A. Ryu and I. Kowalczyk, Long-term kinematic analysis of cervical spine after single-level implantation of Bryan cervical disc prosthesis, The Spine Journal 13 (2013), 628-634.

[3] J. Huppert and J. Beaurain, Comparison between single-and multi-level patients: clinical and radiological outcomes 2 years after cervical disc replacement, European Spine Journal 20 (2011), 1417-1426.

[4] R.C. Sasso and P.A. Anderson, Results of cervical arthroplasty compared with anterior discectomy and fusion: Fouryear clinical outcomes in a prospective, randomized controlled trial, The Journal of Bone and Joint Surgery 93 (2011), 1684-1692.

[5] Z. Jia and Z. Mo, Hybrid surgery for multilevel cervical degenerative disc diseases: A systematic review of biomechanical and clinical evidence, European Spine Journal 23 (2014), 1619-1632.

[6] W.Q. Liu and C.W. Lv, Biomechanical comparison of three-level cervical hybrid contract: an in vitro investigation, Journal of Tsinghua University (Science and Technology) 54 (2014), 685-689.

[7] G.R. Fogel and Z.Y. Li, In vitro evaluation of stiffness and load sharing in a two-level corpectomy: Comparison of static and dynamic cervical plates, The Spine Journal 10 (2010), 417-421.

[8] M.M. Panjabi and J.J. Crisco, Mechanical properties of the human cervical spine as shown by three-dimensional loaddisplacement curves, Spine 26 (2001), 2692-2700.

[9] H.W.D. Hey and C.C. Hong, Is hybrid surgery of the cervical spine a good balance between fusion and arthroplasty? Pilot results from a single surgeon series, European Spine Journal 22 (2013), 116-122.

[10] A. Faizan and V.K. Goel, Adjacent level effects of bi level disc replacement, bi level fusion and disc replacement plus 
fusion in cervical spine-a finite element based study, Clinical Biomechanics 27 (2012), 226-233.

[11] B.W. Cunningham and N. Hu, Biomechanical comparison of single-and two-level cervical arthroplasty versus arthrodesis: effect on adjacent-level spinal kinematics, The Spine Journal 10 (2010), 341-349.

[12] C. Barrey and S. Campana, Cervical disc prosthesis versus arthrodesis using one-level, hybrid and two-level constructs: An in vitro investigation, European Spine Journal 21 (2012), 432-442.

[13] M.J. Lee and M. Dumonski, Disc replacement adjacent to cervical fusion: A biomechanical comparison of hybrid construct versus two-level fusion, Spine 36 (2011), 1932-1939.

[14] S. Martin and A.J. Ghanayem, Kinematics of cervical total disc replacement adjacent to a two-level, straight versus lordotic fusion, Spine 36 (2011), 1359-1366.

[15] M.J. Cardoso and A. Mendelsohn, Cervical hybrid arthroplasty with 2 unique fusion techniques: Technical note, Journal of Neurosurgery: Spine 15 (2011), 48-54.

[16] L. Kang and D. Lin, Artificial disk replacement combined with midlevel ACDF versus multilevel fusion for cervical disk disease involving 3 levels, Orthopedics 36 (2013), e88-94.

[17] Y. Nagamoto and T. Ishii, In vivo three-dimensional kinematics of the cervical spine during head rotation in patients with cervical spondylosis, Spine 36 (2011), 778-783. 